УДК $519.11+512.5$

\title{
Enumerations of Ideals in Niltriangular Subalgebra of Chevalley Algebras
}

\author{
Nikolay D. Hodyunya* \\ Institute of Mathematics and Computer Science \\ Siberian Federal University \\ Svobodny, 79, Krasnoyarsk, 660041 \\ Russia
}

Received 18.11.2017, received in revised form 20.12.2017, accepted 20.02.2018

Let $N \Phi(K)$ be the niltriangular subalgebra of Chevalley algebra over a field $K$ associated with a root system $\Phi$. We consider certain non-associative enveloping algebras for some Lie algebra $N \Phi(K)$. We also study the problem of enumeration of standard ideals in algebra $N \Phi(K)$ over any finite field $K$; for classical Lie types this is the problem which was written earlier (2001).

Keywords: Chevalley algebra, niltriangular subalgebra, enveloping algebra, ideal.

DOI: 10.17516/1997-1397-2018-11-3-271-277.

\section{Introduction}

Any Chevalley algebra over a field $K$ is characterized by a root system $\Phi$ and a Chevalley basis consisting of elements $e_{r}(r \in \Phi)$ and a base of suitable Cartan subalgebra [1, Sec. 4.2]. We fix a positive root system $\Phi^{+} \subseteq \Phi$. The subalgebra $N \Phi(K)$ with the basis $\left\{e_{r} \mid r \in \Phi^{+}\right\}$is said to be a niltriangular subalgebra. In the present paper we consider the following problem.

(A) Find the number of standard ideals of Lie algebra $N \Phi(K)$ over any finite field $K$.

For classical Lie types this problem has arisen earlier as Problem 1 in [2]. In these cases Problem (A) had been solved recently by G. P. Egorychev, V. M. Levchuk, and the author. Standard ideals of a Lie ring $N \Phi(K)$ are distinguished in Sec. 1.

Main Theorem 2.1 in Sec. 2 solves Problem (A) for exceptional Lie types.

Also, we study enveloping algebras of Lie algebras $N \Phi(K)$. According to [3], an algebra $R=(R,+, \cdot)$ (possibly, non-associative) is called an enveloping algebra of a Lie algebra $L$ if $L$ is isomorphic to the algebra $R^{(-)}:=(R,+,[]),,[a, b]:=a b-b a$. (See also Lie-admissible algebras $[4,5]$.) The well-known enveloping algebra $R$ of Lie algebra $N \Phi(K)$ [3, Proposition 1] has also base $\left\{e_{r} \mid r \in \Phi^{+}\right\}$and its choice depends on signs of structural constants of Chevalley basis.

The representation [6] of Lie algebra $N \Phi(K)$ of classical Lie types determines uniquely their enveloping algebra $R$. If $\Phi \neq D_{n}$, then all ideals of such enveloping ring $R$ are exactly standard ideals of Lie ring $N \Phi(K)$. By [3], it is not true for Lie type $D_{n}(n \geqslant 4)$ and, as a corollary, for Lie types $E_{n}(n=6,7,8)$.

As it is shown in the following section, there exist enveloping algebras of type $F_{4}$ both having nonstandard ideals and not having them.

*nkhodyunya@gmail.com

(c) Siberian Federal University. All rights reserved 
We use standard notation from [1]. Let $h t(r)$ be the height of $r \in \Phi$. The highest root in $\Phi^{+}$ is denoted by $\rho$. The Coxeter number $h$ of $\Phi$ is defined by $h t(\rho)+1=h(\Phi)=h$.

\section{Ideals and enveloping algebras of Lie algebra $N \Phi(K)$}

The definition of an enveloping algebra $R$ of an arbitrary finite dimensional Lie algebra $L$ (see Introduction) shows that both algebras can be constructed on the same vector space. Also, every ideal of the enveloping ring $R$ is an ideal of the Lie ring $L$.

In this section we study certain enveloping algebras of a Lie algebra $N \Phi(K)$ and their ideals. According to [1, Proposition 4.2.2], we have

$$
\left[e_{r}, e_{s}\right]=N_{r s} e_{r+s}=-\left[e_{s}, e_{r}\right](r+s \in \Phi), \quad\left[e_{r}, e_{s}\right]=0(r+s \notin \Phi \backslash\{0\}),
$$

where $N_{r s}= \pm 1$ or $|r|=|s|<|r+s|$ and $N_{r s}= \pm 2$ or $\Phi$ is of type $G_{2}$ and $N_{r s}= \pm 2$ or \pm 3 .

Proposition 1.1 ( [3, Proposition 1]). A K-algebra with the basis $\left\{e_{r} \mid r \in \Phi^{+}\right\}$is an enveloping algebra of $N \Phi(K)$ if the product is defined as follows: $e_{r} e_{s}=0$ when $r+s \notin \Phi$, and if $r+s \in \Phi^{+}$ and $N_{r s} \geqslant 1$, then $e_{r} e_{s}=e_{r+s}$ and $e_{s} e_{r}=\left(1-N_{r s}\right) e_{r+s}$.

We distinguish the following ideals in a Lie algebra $N \Phi(K)$ putting on $r \leqslant s \quad\left(r, s \in \Phi^{+}\right)$if $s-r$ is a linear combination of simple roots with nonnegative coefficients:

$$
T(r):=\sum_{r \leqslant s} K e_{s}, \quad Q(r):=\sum_{r<s} K e_{s} .
$$

Roots $r$ and $s$ are called incident ones if $T(r) \subseteq T(s)$ or $T(s) \subseteq T(r)$ (i.e., $s \leqslant r$ or $r \leqslant s$ ). Any set $\mathcal{L}$ of pairwise non-incident roots in $\Phi^{+}$is called a set of corners in $\Phi^{+}$.

If $H \subseteq \sum_{r \in \mathcal{L}} T(r)$ and the inclusion fails under every substitution of $T(r)$ by $Q(r)$, then $\mathcal{L}=\mathcal{L}(H)$ is said to be a set of corners in $H$. By [7], a set $\mathcal{F}(H)$ is said to be a frame of $H$ if

$$
\mathcal{F}(H) \subseteq \sum_{r \in \mathcal{L}} K e_{r}, \quad \mathcal{F}(H)=H \quad \bmod Q(\mathcal{L}) \quad\left(Q(\mathcal{L})=\sum_{r \in \mathcal{L}} Q(r)\right) .
$$

An ideal $H$ of a Lie ring $N \Phi(K)$ is said to be standard if $H=\mathcal{F}(H)+Q(\mathcal{L})$. Evidently, all standard ideals of Lie ring $N \Phi(K)$ are ideals of any enveloping ring $R$ from Proposition 1.1

The representation [6] of Lie algebras $N \Phi(K)$ of classical Lie types determines uniquely their enveloping algebra $R$. All ideals of such enveloping $\operatorname{ring} R$ for $\Phi \neq D_{n}$ are exactly standard ideals of Lie ring $N \Phi(K)$. By [3], it is not true for Lie type $D_{n}(n \geqslant 4)$ and also, as a corollary, for Lie types $E_{n}(n=6,7,8)$. We now show that both cases are possible for Lie type $F_{4}$.

Theorem 1.1. For Lie type $F_{4}$ Proposition 1.1 allows to construct enveloping algebras $R_{1}$ having nonstandard ideals, and $R_{2}$ in which all ideals are standard.

Proof. Note that the enveloping algebra $R$ from Proposition 1.1 depends on choice of signs of structural constants $N_{\text {rs }}$.

Similarly to [1, Lemma 5.3.1], we use an ordering $\prec$ on the space containing roots $\Phi$ such that $r \prec s$ implies $h(r) \leqslant h(s)$. An ordered pair $(r, s)$ of roots is called a special pair if $r+s \in \Phi$ and $0 \prec r \prec s$. An ordered pair $(r, s)$ is called extraspecial if $(r, s)$ is a special pair and if for all special pairs $\left(r^{\prime}, s^{\prime}\right)$ with $r+s=r^{\prime}+s^{\prime}$ we have $r \preccurlyeq r^{\prime}$.

Proposition 1.2. The signs of the structure constants $N_{r s}$ may be chosen arbitrarily for extraspecial pairs $(r, s)$, and then the structure constants for all pairs are uniquely determined. 
Proof. See [1, Proposition 4.2.2].

For the root system $\Phi$ of type $F_{4}$, we need notation from [8]. The positive root systems of types $B_{n}$ and $C_{n}$ [9, Tables I-IV] can be written, respectively, as

$$
\begin{gathered}
C_{n}^{+}=\left\{p_{i v}|0<| v \mid \leqslant i \leqslant n, v \neq i\right\}, \quad p_{i, m j}=\epsilon_{i}-m \epsilon_{j}, \quad 1 \leqslant j \leqslant i \leqslant n, m=0,1,-1 ; \\
B_{n}^{+}=\left\{q_{i j}|0 \leqslant| j \mid<i \leqslant n\right\}, \quad q_{i, m j}=\epsilon_{i}-m \epsilon_{j} .
\end{gathered}
$$

Then the positive system $F_{4}^{+}$is represented as the union $C_{4}^{+} \cup B_{4}^{+}$with the given intersection

$$
B_{4}^{+} \cap C_{4}^{+}=\left\{q_{i 0}, p_{i,-i}(1 \leqslant i \leqslant 4)\right\} .
$$

Also, we use the following diagram from [8]. (The roots are accompanied by the notation (abcd) from [9, Table VIII].)

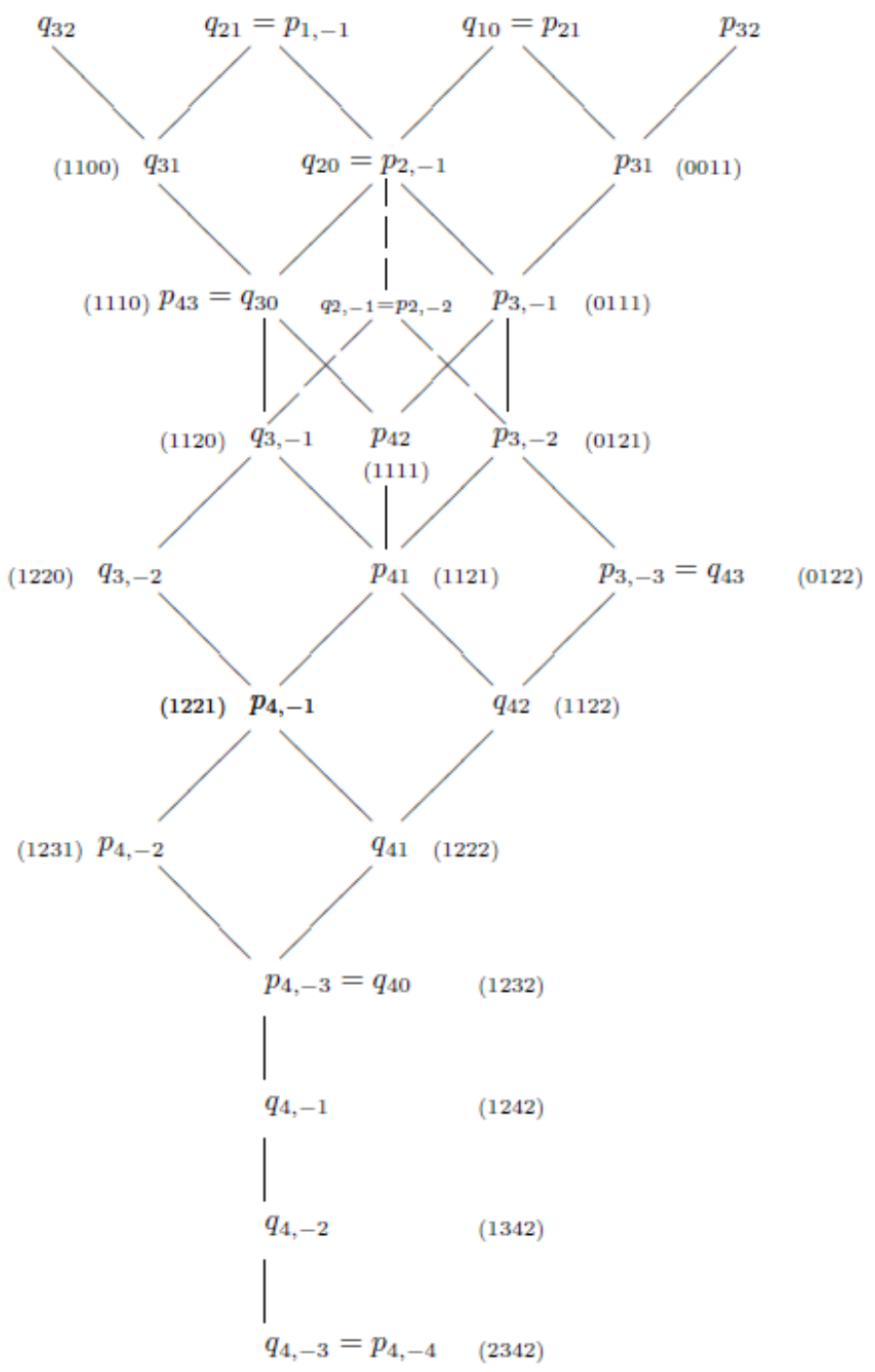

Fig. 1. The positive roots of the system $F_{4}$ 
The relation $q_{32} \prec q_{21} \prec q_{10} \prec p_{32}$ of simple roots determines uniquely the ordering $\prec$ (Fig. 1). Using Proposition 1.1 choose an arbitrary enveloping algebra $R$ for Lie algebra $N \Phi(K)$ of type $F_{4}$. Recall that an ideal $H$ of $R$ is standard iff $Q(r) \subseteq H$ for all $r \in \mathcal{L}(H)$.

It is clear that if $M$ is a subset in an ideal $H$ of $R$ and $\mathcal{F}(M)=K e_{r}$, then $T(r) \subseteq H$. Let $L_{i}=\sum_{i \geqslant h(r)} T(r), 1 \leqslant i<h$. It is not difficult to prove the following lemma.

Lemma 1.1. Every ideal $H \subseteq L_{4}$ in the enveloping ring $R$ is standard.

Now we construct algebras $R_{i}$ from Theorem 1.1. Assume $N(r, s):=N_{r s}$, and also,

$$
\begin{gathered}
N\left(q_{21}, q_{3,-1}\right)=1, \quad N\left(q_{21}, p_{31}\right)=-1, \quad N\left(q_{32}, p_{3,-1}\right)=1, \quad N\left(q_{10}, p_{3,-1}\right)=-1, \\
N\left(q_{32}, q_{2,-1}\right)=-1, \quad N\left(q_{32}, q_{21}\right)=-1, \quad N\left(q_{10}, p_{32}\right)=-1, \quad N\left(q_{21}, q_{10}\right)=-1 .
\end{gathered}
$$

For algebra $R_{1}$ we additionally set $N\left(q_{32}, q_{20}\right)=-1, N\left(q_{10}, q_{20}\right)=2$, and

$$
N\left(p_{32}, q_{2,-1}\right)=-1, \quad N\left(p_{32}, q_{30}\right)=-1, \quad N\left(q_{10}, q_{31}\right)=1 .
$$

By choosing arbitrarily the remaining structural constants $N_{r s}$ we obtain the algebra $R_{1}$. One can see that ideals in the algebra $R_{1}$ of the form

$$
K\left(e_{q_{30}}+c e_{q_{2,-1}}\right)+K\left(e_{p_{42}}+c e_{p_{3,-2}}\right)+T\left(q_{3,-1}\right)+T\left(q_{43}\right) \quad\left(c \in K^{*}\right)
$$

are nonstandard. Moreover, It can easily be checked that all other ideals in the algebra $R_{1}$ are standard.

Lemma 1.2. The algebra $R_{1}$ has nonstandrd ideals and they are exhausted by ideals (2).

Further, we use the following lemma to construct algebra $R_{2}$ which is not isomorphic to $R_{1}$.

Lemma 1.3. All ideals in the ring $R$ are standard if the following equalities are satisfied:

$$
\begin{array}{cl}
N\left(q_{21}, q_{3,-1}\right)=-N\left(q_{21}, p_{31}\right), & N\left(q_{32}, p_{3,-1}\right)=-N\left(q_{32}, q_{2,-1}\right), \\
N\left(q_{10}, p_{3,-1}\right)=-N\left(q_{10}, q_{31}\right), & N\left(p_{32}, q_{30}\right)=-N\left(p_{32}, q_{2,-1}\right), \\
N\left(q_{21}, p_{31}\right)=N\left(q_{32}, q_{21}\right), & N\left(q_{10}, q_{31}\right)=-N\left(q_{10}, p_{32}\right), \\
N\left(q_{32}, q_{21}\right)=N\left(q_{21}, q_{10}\right), & N\left(q_{21}, q_{10}\right)=N\left(q_{10}, p_{32}\right) .
\end{array}
$$

Proof. The proof is by direct calculation.

To construct algebra $R_{2}$, as before, assume (1). Also set $N\left(q_{32}, q_{20}\right)=-1$ and $N\left(q_{10}, q_{20}\right)=-2$. Then, by the Jacobi identity, $N\left(p_{32}, q_{20}\right)=1$ and

$$
N\left(p_{32}, q_{2,-1}\right)=1, \quad N\left(p_{32}, q_{30}\right)=-1, \quad N\left(q_{10}, q_{31}\right)=1 .
$$

By choosing arbitrarily the remaining structural constants $N_{r s}$ we obtain the algebra $R_{2}$.

Lemma 1.4. All ideals in the algebra $R_{2}$ are standard.

Finally, by combining Lemmas 1.2 and 1.4 we prove Theorem 1.1. 


\section{The completion of problem's (A) solution}

Denote by $N \Phi(q)$ the algebra $N \Phi(K)$ over finite field $K=G F(q)$. Problem (A) of enumeration of standard ideals in Lie algebras $N \Phi(q)$ had been recently solved for classical Lie types (as Problem 1 in [2]) by G.P. Egorychev, V. M. Levchuk, and the author. In these section we complete the solution of Problem (A).

The following theorem gives the solution of Problem (A) for exceptional Lie types.

Theorem 2.1. The number of standard ideals of a Lie algebra $N \Phi(q)$ of exceptional Lie type is equal to

$$
\begin{aligned}
G_{2}: & q+7 \\
F_{4}: & q^{4}+3 q^{3}+44 q^{2}+32 q+25 \\
E_{6}: & q^{9}+3 q^{8}+4 q^{7}+67 q^{6}+69 q^{5}+230 q^{4}+306 q^{3}+94 q^{2}+22 q+37 \\
E_{7}: & 2\left(q^{12}+q^{11}+3 q^{10}+32 q^{9}+90 q^{8}+118 q^{7}+394 q^{6}+449 q^{5}+\right. \\
\quad & \left.+708 q^{4}+300 q^{3}-79 q^{2}+31 q+32\right) ; \\
E_{8}: & q^{16}+3 q^{15}+4 q^{14}+7 q^{13}+237 q^{12}+239 q^{11}+693 q^{10}+1647 q^{9}+3554 q^{8}+ \\
& \quad+4283 q^{7}+5829 q^{6}+7055 q^{5}+3773 q^{4}-2361 q^{3}-244 q^{2}+239 q+121 .
\end{aligned}
$$

Proof. We need the following definition. A subspace $S$ of the space $K^{m}$ is called m-proper if for all $i, 1 \leqslant i \leqslant m$, there exists an element $\left(a_{1}, \ldots, a_{m}\right) \in S$ such that $a_{i} \neq 0$.

Similarly to Section 1, every standard ideal $H$ of Lie algebra $N \Phi(q)$ is characterized by a set of corners $\mathcal{L}(H)=\left\{r_{1}, r_{2}, \ldots, r_{m}\right\}$ and a frame $\mathcal{F}(H)$. So, to each standard ideal $H$ there corresponds a unique pair $(\mathcal{L}, S)$ such that $H$ is equal to the ideal

$$
H(\mathcal{L}, S)=Q(\mathcal{L})+\left\{a_{1} e_{r_{1}}+a_{2} e_{r_{2}}+\cdots+a_{m} e_{r_{m}} \mid\left(a_{1}, a_{2}, \ldots, a_{m}\right) \in S\right\} .
$$

The second term in $(3)$ is a frame of the ideal $H(\mathcal{L}, S)$. This yields that the enumeration of standard ideals coincides with the enumeration of ideals of the form (3). Denote by $\tilde{V}_{m, t}$ the number of all $m$-proper $t$-dimensional subspaces in $K^{m}$ and by $B(\Phi, m)$ denote the number of sets of corners $\mathcal{L}$ in $\Phi^{+}$with $|\mathcal{L}|=m$. From the established one-to-one correspondence between standard ideals and pairs $(\mathcal{L}, S)$, we obtain the following

Lemma 2.1. The number of standard ideals in the algebra $N \Phi(q)$ of Lie rank $n$ is

$$
\Omega(\Phi, q)=1+\sum_{m=1}^{n} B(\Phi, m) \sum_{t=1}^{m} \tilde{V}_{m, t} .
$$

Besides the solution of Problem 1 for type $A_{n},[10]$ provides the formula

$$
\widetilde{V}_{m, t}=\sum_{1=j_{1}<j_{2}<\ldots<j_{t} \leqslant m} \frac{\left(q^{t}-1\right)^{m-j_{t}}}{(q-1)^{t-j_{t}}} \cdot \prod_{k=2}^{t-1}\left(\frac{q^{k}-1}{q-1}\right)^{j_{k+1}-j_{k}-1} \quad(1 \leqslant t \leqslant m) .
$$

In his paper [11], G. P. Egorychev has found a simpler form of this formula.

Lemma 2.2 ( [11, Lemma 4]). The number of m-proper $t$-dimensional subspaces of the space $K^{m}$ over the finite field $K=G F(q)$ is

$$
\widetilde{V}_{m, t}=\sum_{k=0}^{m-t}(-1)^{m-t-k} q^{k}\left(\begin{array}{c}
m-1 \\
t+k-1
\end{array}\right)\left[\begin{array}{c}
t+k-1 \\
k
\end{array}\right]_{q} .
$$


By using Lemma 2.1 we immediately obtain $\Omega(\Phi, q)=q+7$ for type $G_{2}$. In the remaining cases, we obtain the numbers $B(\Phi, m)$ by using the representations of $\Phi^{+}$of type $F_{4}$ in [8] and of types $E_{n}(n=6,7,8)$ in [12]. Tab. 1 represents the results of computations. (See also [13, Remark 5.2].)

Substituting the corresponding values of Tab. 1 and (5) for $B(\Phi, m)$ and $\widetilde{V}_{m, t}$ in (4), we prove Theorem 2.1.

Table 1. The values of $B(\Phi, m)$ for types $F_{4}$ and $E_{n}$

\begin{tabular}{|c|c|c|c|c|c|c|c|c|c|}
\hline$\Phi / \mathrm{m}$ & 0 & 1 & 2 & 3 & 4 & 5 & 6 & 7 & 8 \\
\hline$F_{4}$ & 1 & 24 & 55 & 24 & 1 & & & & \\
\hline$E_{6}$ & 1 & 36 & 204 & 351 & 204 & 36 & 1 & & \\
\hline$E_{7}$ & 1 & 63 & 546 & 1470 & 1470 & 546 & 63 & 1 & \\
\hline$E_{8}$ & 1 & 120 & 1540 & 6120 & 9518 & 6120 & 1540 & 120 & 1 \\
\hline
\end{tabular}

The author expresses his gratitude to V. M. Levchuk for guidance and important corrections. The author was supported by RFBR (project no. 16-01-00707).

\section{References}

[1] R.Carter, Simple Groups of Lie type, Wiley and Sons, New York, 1972.

[2] G.P.Egorychev, V.M.Levchuk, Enumeration in the Chevalley algebras, ACM SIGSAM Bulletin, 35(2001), no. 2, 20-34.

[3] V.M. Levchuk, Niltriangular subalgebra of Chevalley algebra: the enveloping algebra, ideals and automorphisms, Dokl. Math., 478(2018), no. 2.

[4] A.Albert, Power-Associative Rings, Trans. Amer. Math. Soc., 64(1948), no. 3, 552-593.

[5] H.C.Myung, Some Classes of Flexible Lie-Admissible Algebras, Trans. Amer. Math. Soc., 167(1972), 79-88.

[6] V.M.Levchuk, Automorphisms of unipotent subgroups of Chevalley groups, Algebra and Logic, 29(1990), no. 3, 211-224.

[7] V.M.Levchuk, G.S.Suleimanova, Extremal and maximal normal abelian subgroups of a maximal unipotent subgroup in groups of Lie type, J. Algebra, 349(2012), no. 1, 98-116.

[8] V.M.Levchuk, Automorphisms of unipotent subgroups of Lie type groups of small ranks, Algebra and Logic, 29(1990), no. 2, 97-112.

[9] N.Bourbaki, Groupes et algebres de Lie, Chapt. IV-VI, Hermann, Paris, 1968.

[10] V.P.Krivokolesko, V.M.Levchuk, Enumeration of ideals in exceptional nilpotent matrix algebras, Trudy IMM UrO RAN, 21(2015), no. 1, 166-171 (in Russian).

[11] G.P.Egorychev, Enumeration of proper $t$-dimensional subspaces of the space $V_{m}$ over a field GF(q), Izv. Irkutsk. GU, ser. matem., 17(2016), no. 3, 12-22 (in Russian). 
[12] G.S.Suleimanova, Doctor's Dissertation in Mathematics and Physics, SFU, Krasnoyarsk, 2013 (in Russian).

[13] C.A.Athanasiadis, On a refinement of the generalized Catalan numbers for Weyl groups, Transact. of Amer. Math. Soc., 357(2005), no. 1, 179-197.

\section{Перечисления идеалов в нильтреугольной подалгебре алгебры Шевалле}

Николай Д. Ходюня

Институт математики и фундаментальной информатики Сибирский федеральный университет Свободный, 79, Красноярск, 660041

Россия

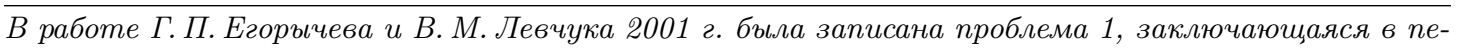
речислении стандартных идеалов нильтреугольных подалгебр $N \Phi(G F(q))$ алгебр Шевалле классических типов. Мы решаем аналог проблемы 1 для исключительных типов. С помощью недавно введенной конструкиии В. М. Левчука обертывающих алгебр для $N \Phi(K)$ исключительного типа $F_{4}$ найдены обертывающие алгебры как с нестандартными иделами, так и без них.

Ключевые слова: алгебра Шевалле, нильтреугольная подалгебра, обертывающая алгебра, идеал. 